\title{
Energy Efficient Communication for Underwater Wireless Sensors Networks
}

\author{
Ammar Babiker and Nordin Zakaria \\ PETRONAS University of Technology \\ Malaysia
}

\section{Introduction}

Water covers more than $70 \%$ of the planet, contains much of its natural resources, and defines the greater territories of many nations. With the increasing use of underwater sensors for the exploitation and monitoring of vast underwater resources, underwater wireless sensor network (UWSN), mostly based on acoustic transmission technologies, have been developing steadily in terms of operation range and data throughput.

As in terrestrial sensor networks, various data transport protocols have been designed for UWSN (Pompili, 2007). However, as yet, there is no internationally accepted standard for underwater communication. The lack of standard is due to the technical challenges that still persist in establishing reliable underwater wireless data communication. Firstly, in underwater environment, electromagnetic wave is rarely of use, unlike in terrestrial space, as it can only travel a short distances due attenuation and absorptions effects. Optical signal suffers from scattering and absorption in underwater (Akyildiz, et al., 2005). Hence, to date acoustic energy is the most widely used type of signals used in underwater data transmission. Secondly, the fluctuating nature of ocean condition causes high bit error rate in acoustic transmission. Underwater acoustic transmission is also affected by path loss caused by spreading and absorption, noise which comes from many sources like water current, rain, wind, seismic and volcanic activities or biological phenomena (Pompili, 2007). Signal reflection and refraction from the surface and seabed, topographic sources like hills and hollows are some example error sources.

Hence in underwater environment, the two main issues of concern are namely: reliability and energy efficiency. These two issues are inter-twined. Reliability requires errorcorrection, and error-correction requires energy. More reliability tends to imply higher energy consumption, causing difficulty in applications that require nodes to be operated underwater for long periods of time without batteries recharging, and in aquatic environments that render hard the task of recharging or replacing batteries (Pompili, 2007; Preisig, 2007). Appropriate strategy must therefore be in-place to ensure reliable data transmission, while conserving energy.

In this chapter, we focus on the energy efficiency issue in UWSN. We develop a mathematical model of the efficiency of acoustic data communication in realistic underwater environment. We analyze existing error-correction techniques, and we then propose a new adaptive hybrid error correction technique that improves upon existing techniques. 
We organize the rest of this chapter as follows: In section 2, we briefly review the basics of acoustic propagation. In section 3 , we review the two most widely used underwater error correction techniques, ARQ and FEC. In section 4, we present mathematical and simulation analysis of the energy efficiency of the techniques. In section 5, an Adaptive Hybrid Energy Efficient Error Correction (AHEC) technique for Underwater Wireless Sensor Networks (UWSN) data transmission is presented. Finally, in section 6, we conclude the chapter.

\section{Basics of acoustic propagation}

Factors that characterized underwater acoustic propagation include path loss, noise, multipath, Doppler spread, and high and variable propagation delay. Those factors are the main reason for the variability in the acoustic channel. Bandwidth varies from a few $\mathrm{KHz}$ in a long range system which operates over several tens of kilometers to more than hundred $\mathrm{KHz}$ in a short-range system that operates over several tens of meters. UAC system are classified according to their communication ranges as shown in Table 1 (Akyildiz, et al., 2004, 2005):

\begin{tabular}{|c|c|c|}
\hline & Range $(\mathrm{Km})$ & Bandwidth $(\mathrm{KHz})$ \\
\hline Very long & 1000 & $<1$ \\
\hline Long & $10-1000$ & $2-5$ \\
\hline Medium & $1-10$ & $=10$ \\
\hline Short & $0.1-1$ & $20-50$ \\
\hline Very short & $<0.1$ & $>100$ \\
\hline
\end{tabular}

Table 1. Underwater Acoustic Communication System Ranges

Below are the factors that characterize underwater acoustic propagation (Colin et al., 2007; Joshy and Babu, 2010; Stojanovic and Preisig, 2009):

- Path loss: there are two main sources for path losses for underwater acoustic propagation:

- Attenuation: this is the loss due to the conversion of acoustic energy into heat which known as absorption loss.

- Spreading: this is the loss due to the expansion of the signal energy over a large area as the wave propagates forward.

- $\quad$ Noise: there are two kind of noise:

- Man-made noise: it is caused by machinery (pumps, reduction gears, etc), shipping activities, etc.

- Ambient noise: this is caused by the movement of water which includes tides, current, storms, wind, and rain. It is also caused by biological phenomena. Ambient noise depends mainly on frequency, so it must be considered when selecting frequency band in underwater communications systems (Preisig , 2007).

- Multipath: In most environments, the ocean can be modelled as a wave guide for communication signals. This waveguide is characterized by a reflecting surface and ocean bottom and a variant sound speed. Reflection, refraction and diffraction will occur with those surfaces resulting in multiple propagation paths from the source to the 
receiver. Horizontal channel is characterized by long multipath spreads compared with vertical one. A multipath with varying impulse response tends to be subjected to an Inter Symbol Interference (ISI) that causes severe degradation in the acoustic signals.

- High delay and delay variance: underwater acoustic signal speed is just $1500 \mathrm{~m} / \mathrm{s}$, which is lower than electromagnetic signal by more than 5 orders of magnitude. The propagation delay is large too (about $0.67 \mathrm{~s} / \mathrm{km}$ ).

- Doppler spread: it is significant in underwater acoustic channel, and cause degradation in the performance of digital communications..

Most of the factors mentioned above are caused by the chemical-physical properties of the sea water such as temperature, salinity and density, which these factors vary with depth, space and time.

\section{Error correction techniques}

Automatic Repeat reQuest (ARQ) and Forward Error Correction (FEC) are two commonly used strategies to combat error in underwater transmission (Bin et al, 2008). ARQ which proposes retransmission (Kunal et al., 2010), are widely used in data communications system for error control as they are simple and provide high reliability. However, the throughput is not constant and decreases rapidly in high bit error rate cases (Lin et al., 1984). In FEC, redundancy is added for error prevention. Redundant bits are encapsulated with data bits to form encoded information. However this increases the payload for transmission. Addition of redundant bit is known as channel coding. Error Correcting Codes (ECC) (block or convolutional) are used for this purpose. FEC codes have constant throughput which is equal to the code rate. However it has the drawback of using parity bit irrespective of the existence of errors. Reliability can be enhanced by combining FEC and ARQ, forming what is known as Hybrid-ARQ (HARQ) (Kunal et al., 2010).

\subsection{Automatic repeat request}

ARQ uses error detection codes, acknowledgement and/or negative acknowledgement messages, and time out to retransmit error packet. The basic idea is that the transmitter after sending the packet waits for specific time (time out) to receive an acknowledgement. If it receives positive acknowledgement (ACK), it sends the next packet. On the other hand, if it receives negative acknowledge (NAC) or timed out before receiving any acknowledgement, it then retransmits the same packet. The process repeats until an ACK has been received by the transmitter or a specific number of retransmission has been reached.

In (Tan et al., 2007), an opportunistic (hybrid implicit/explicit) acknowledgement scheme suitable for stop and wait protocols in underwater is proposed. The simple stop and wait (S $\& W)$ protocol is chosen as it is the most popular method in underwater acoustic communication due to the half-duplex property of acoustic modem. In the context of a multi hop channel, the work in (Tan et al., 2007) proposed that the acknowledgement can be achieved explicitly by transmitting an acknowledgement packet per successfully received packet, or implicitly by making use of the broadcast nature of the medium.

In (Lee et al., 2008), the channel sharing property inherent in underwater environment is utilized in proposing an efficient ARQ scheme. In this scheme packet size is controlled in such a way that transmission time becomes smaller than propagation delay. Collision free 
transmission between multiple nodes is achieved by scheduling packets. In a multiple hop setup, the acknowledgement packet is replaced by overhearing packet transmitted from next hop. Overhearing as an acknowledgement method not only saves energy but it also minimizes overhead and transmission latency. The scheme is evaluated by comparing it with an existing stop and wait ARQ in term of the latency, and it shows a reduction in the latency. The latency and energy efficiency is still a problem in bad channel conditions cases though.

In (Gao et al., 2009), the authors make use of the long propagation delay in underwater environment to transmit and receive in a juggling manner. This juggling scheme enables a continuous ARQ to be implemented irrespective of the half-duplex property of the acoustic modem. This scheme decreases the propagation time by having more than one packet in the channel between transmitter and receiver. This leads to high throughput compared with the other variant ARQ schemes, but it is still unsuitable in bad channel conditions or in a longer distance ranges.

In (Valera et al. 2009), a modular and lightweight of an opportunistic multi-hop ARQ (Tan et al., 2007) was implemented for real system. An extensible network stack suitable for challenged underwater acoustic networks was designed and implemented in the work. Evaluation demonstrated that the opportunistic ARQ can provide significant improvement in terms of data delivery ratio. The disadvantage of this technique is an increase in end-toend delay due to queuing and retransmissions.

\subsection{Forward error correction technique}

Forward Error Correction (FEC) or error control coding is a system for achieving reliable message transmission in a communication system by correcting errors in the receiver side (hence the name 'Forward').

Recent and major activities on error control coding can be summarized as follows:

- Research on good structural properties, and high error correcting performance.

- Efficient encoding and decoding strategies.

- Applicability of coding in various transmission system and channels.

Forward error correction can be used in two levels, namely at the bit and the byte level. Bit level correction is achieved by adding redundant bits to the data in the sender. At the packet level, additional check packets are transmitted to help recover lost packets. In the FEC, no back channel is needed, but high bandwidth is required. It is therefore suitable in cases where retransmission is costly or impossible, as in broadcasting. The numbers of errors which can be corrected depend on the code rate and the type of coding used. Therefore, different FEC codes are suitable for different conditions.

There are two main types of FEC; the first one is the block codes which work on a fixed-size blocks (packets of bits or symbols), the most famous block codes are Reed-Solemn, Golay, (Bose, Chandhuri and Hocquenghem) $\mathrm{BCH}$ code, multidimensional parity and Hamming codes. The other type of FEC is convolutional codes, which work on bit or symbol streams of arbitrary length. It is often decoded using Viterbi algorithm, and it can be turned into block code if desired. 
Most telecommunication systems use fixed types of FEC code, which is designed for the expected worst case bit error rate. These codes will fail if the bit error rate ever gets worse.

In (Guo, 2006), error recovery through network coding was explored for underwater sensor networks. The computational power of underwater sensors along with the multiple routes provided by the broadcast nature of acoustic medium are the main reasons for applying network coding. In this technique the source and intermediate nodes encode packets and send them on multiple routes. The packets are then recovered in the destination by combining packets from different routes.

In (Xie and Cui, 2007), the Segmented Data Reliable Transport protocol (SDRT) is proposed,. The protocol is a hybrid of FEC and ARQ. It sends data block by block and hop by hop. The sender encodes the packet using erasure codes, and sends it to an intermediate node. The intermediate node reconstructs the packet and encodes it and sends it to the next hop. The sender continues to send the data until it receive an acknowledgement from its next node, and this is the main problem with SDRT as it wastes energy. SDRT however improves channel utilization and simplify protocol management.

In ( Liu et al., 2010; Bin et al., 2008), the Internode distance-based Redundancy Reliable Transport Protocol (ARRTP) is proposed. It is a hybrid of two types of error correction techniques which encode message on bit and/or packet level. ARRTP is based on distance as adaptation factor. For each range of distance, one or a hybrid of two techniques is used. The technique was also investigated in cooperation mode, making use of the broadcast nature of acoustic signal. ARRTP is found to have better probability of success and energy efficient in single and multi-transmission. This technique is based on fixed channel conditions analysis, so it is unsuitable in variable underwater channel conditions.

\section{Transmission energy efficiency mathematical and simulation analysis}

Underwater acoustic channel are characterized by variable channel conditions and variable distances between sensor nodes due to water currents. As said earlier in the chapter, in such situations, reliable and efficient communication data transport is needed. Reliability is usually achieved by using error correction techniques. However, energy consumption needs to be considered as it is difficult to recharge or even replace batteries for a large number and sparsely distributed sensors. This condition is even worse in underwater due to the harsh aquatic medium (Colin, 2007; Xie and Cui, 2007). Hence, the design of error correction techniques should take into consideration the energy conservation requirement.

In this section, we first develop a model for underwater propagation. A mathematical analysis for energy efficiency for FEC and ARQ techniques in underwater environment is then presented. The analysis is based on communication distance and packet size, and considers the effects of wind speed, and shipping factor. Simulation was done using MATLAB to validate the mathematical analysis results. Results depicting the energy efficiencies of transmission using ARQ and FEC for different packet size, different distances, and different channel conditions (wind speed and shipping factors) are presented.

\subsection{Underwater propagation model}

The propagation model is responsible for calculating the SNR at the receiver after attenuation and noise are taken into account. To calculate the SNR at the receiver, both the 
attenuation of the acoustic signal in water and the ambient noise need to be calculated. The total attenuation is calculated based on the spreading losses and Thorp approximation for the absorption loss (Urick, 1983; Yang and Liu, 2009; Liu et al., 2010; Harris and Zorzi, 2007).

\subsubsection{Attenuation}

Attenuation consists of two parts, the first one is the absorption loss and the second part is the spreading loss. To calculate the absorption loss at a given frequency, Thorp's approximation function divides the frequencies into two groups; one group under $400 \mathrm{~Hz}$ and the other one over $400 \mathrm{~Hz}$ as follows:

$$
\begin{aligned}
10 \log a(f)= & 0.11 \frac{f^{2}}{1+f^{2}}+44 \frac{f^{2}}{4200+f}+2.75 \times 10^{-4} f^{2}+0.003 \mathrm{f}>0.4 \\
& =0.002+0.11 \times\left(\frac{f}{1+f}\right)+0.011 f \mathrm{f}<0.4
\end{aligned}
$$

where $\mathrm{a}(\mathrm{f})$ is given in $\mathrm{dB} / \mathrm{km}$ and $\mathrm{f}$ in $\mathrm{KHz}$ for underwater communications. Combining absorption effects and spreading loss, the total attenuation is as follows:

$$
10 \log A(l, f)=k \log l+l \times 10 \log a(f)
$$

where the first term is the spreading loss and the second term is the absorption loss. The spreading coefficient $\mathrm{k}$ defines the geometry of the propagation (i.e., $\mathrm{k}=1$ for cylindrical propagation (shallow water), $\mathrm{k}=2$ for spherical propagation (deep water), and $\mathrm{k}=1.5$ for practical spreading) (Urick, 1983).

\subsubsection{Noise}

The background noise in ocean has many sources which vary with frequency and location (Wenz and Gordon, 1939). The following formulas give the power spectral density of the four noise components (Yang and Liu, 2009; Liu, 2010; Harris and Zorzi, 2007; Webb, 1992):

$$
\begin{gathered}
10 \log N_{t}(f)=17-30 \log (f) \\
10 \log N_{s}(f)=40+20(s-0.5)+26 \log (f)-60 \log (f+0.03) \\
10 \log N_{w}(f)=50+7.5 \times w^{0.5}+20 \log (f)-40 \log (f+0.4) \\
10 \log N_{t h}(f)=-15+20 \log (f)
\end{gathered}
$$

Where $N_{t}$ is the noise due to turbulence, $N_{s}$ is the noise due to shipping (the shipping variable s take the values between 0 and 1), $\mathrm{N}_{\mathrm{w}}$ is the noise due to wind (the wind variables $\mathrm{w}$ represent wind speed in $\mathrm{m} / \mathrm{s}$ ), and $\mathrm{N}_{\text {th }}$ represents thermal noise. The overall noise power spectral density for a given frequency $\mathrm{f}(\mathrm{KHz})$ is then:

$$
N(f)=N_{t}(f)+N_{s}(f)+N_{w}(f)+N_{t h}(f)
$$




\subsubsection{Signal to noise ratio}

It is well known that SNR of an emitted underwater signal at the receiver is given by (Yang and B. Liu, 2009; Harris and M. Zorzi, 2007; Brekhovskikh and Lysanov, L.1982)

$$
S N R=S L-A(l, f)-N(f)-D I
$$

where $\mathrm{N}(\mathrm{f}), \mathrm{A}(\mathrm{l}, \mathrm{f})$ are in $\mathrm{dBs}$ given from equations (2) and (7). Assuming Omni-directional directivity, directivity index $(\mathrm{DI})=0$. The source level $S L=20 \log I / 1 \mu \mathrm{Pa}$, where I is the intensity at $1 \mathrm{~m}$ from the source in watt/ $\mathrm{m} 2$, given by:

$$
I=\frac{P_{t}}{2 \pi H}
$$

Where $\mathrm{P}_{\mathrm{t}}$ is the transmission power, and $\mathrm{H}$ is the water depth in $\mathrm{m}$.

\subsection{Energy efficiency mathematical analysis}

The data packet format in ARQ case can be presented as in Figure 1 (a). It consists of a header field a bits long, payload of size $n$ bits and a Frame Check Sequence (FCS) $\tau$ bits long. The acknowledgement packet length is ack.

In FEC case it can be presented as in Figure 1 (b). It consists of a payload of size (n-k) bits long, a parity check of $\mathrm{k}$ bits and a header field a bits long.

\begin{tabular}{|c|c|c|}
\hline Header & FCS & Payload \\
\hline $\mathrm{a}$ & $\tau$ & $n$ \\
\hline
\end{tabular}

Fig. 1. (a): ARQ Packet Format

\begin{tabular}{|c|c|c|}
\hline Header & Parity check & Payload \\
\hline $\mathrm{a}$ & $\mathrm{k}$ & $\mathrm{n}-\mathrm{k}$ \\
\hline
\end{tabular}

Fig. 1. (b): FEC Packet Format

\subsubsection{Optimization metric}

Energy efficiency is the suitable metric which captures both energy and reliability constraints, and it is defined as (Sankarasubramaniam et al, 2003; Tian et al. , 2008):

$$
\begin{gathered}
\eta=\eta_{e}(1-P E R) \\
=\frac{E_{e f f}}{E_{t o t}}(1-P E R)
\end{gathered}
$$

Where $\eta$ is the energy efficiency, $\eta_{e}$ is the energy throughput, $\mathrm{r}=(1-\mathrm{PER})$ is the Packet Acceptance Rate (PAR), which accounts for data reliability, and $\frac{E_{\text {eff }}}{E_{\text {tot }}}$ denotes the energy 
throughput. Therefore, the energy efficiency $\eta$ represents the useful fraction of the total energy expenditure in a communication link between sensors.

\subsubsection{Bit error rate calculation}

Using 8-Phase Shift Keying (PSK) scheme as the suitable modulation techniques for underwater acoustic communication, the symbol error probability $\mathrm{P}_{\mathrm{s}}$ for ARQ is given by (Labrador et al., 2009):

$$
P_{s} \approx 2 Q\left(\sqrt{2 \gamma_{s}} \sin \frac{\pi}{M}\right.
$$

where $\mathrm{M}=8$ for 8 -PSK, and the bit error probability $\mathrm{P}_{\mathrm{b}}$ is given by:

$$
P_{b}=P_{s} / 3
$$

Whereas for FEC convolution code (Lee et al., 2008):

$$
P_{b}=\frac{1}{k} \sum_{d=d_{\text {free }}}^{\infty} w(d) Q\left(\sqrt{2 d R_{c} \gamma_{b}}\right.
$$

where $\mathrm{w}(\mathrm{d})$ is the weight distribution function, dfree is the minimum hamming distance, and $\gamma_{b}$ is the received SNR, $R_{c}=k / k+1$ is the code rate.

\subsubsection{ARQ energy efficiency mathematical analysis}

Energy consumption of sensor node for communication in one hop is given by:

$$
E_{A R Q}=E_{A R Q}^{t r}+E_{A R Q}^{r e}
$$

Where $E_{A R Q}^{t r}$ is the energy consumed by the sender in transmitting the data and receiving the acknowledgement, and $E_{A R Q}^{r e}$ is the energy consumed by the receiver in receiving the data and transmitting the acknowledgement as presented in the following equations:

$$
\begin{aligned}
& E_{A R Q}^{t r}=E_{\text {data }}^{t r}+E_{a c k}^{r e} \\
= & P_{t r} l_{\text {data }} T_{t r}+P_{r e} l_{a c k} T_{t r} \\
& E_{A R Q}^{r e}=E_{\text {data }}^{r e}+E_{a c k}^{t r} \\
= & P_{r e} l_{\text {data }} T_{t r}+P_{t r} l_{a c k} T_{t r}
\end{aligned}
$$

Where $P_{t r / r e}$ is the power consumed in transmitting/ receiving, and $T_{t r}=1 / R$ is the time of transmitting 1 bit. From Figure 1 (a), using the bit error rate probability $P_{b}$ in (12), and 
assuming independent bit errors, the Packer Error Rate (PER) for ARQ can be derived as follows:

$$
\operatorname{PER}_{A R Q}=1-\left(1-P_{b}\right)^{n+\alpha+\tau}
$$

This expression closely approximates PER under bursty error conditions.

From equation (9) energy efficiency of ARQ without retransmission strategy can hence be written as:

$$
\begin{gathered}
E_{A R Q}=\frac{E_{A R Q}^{e f f}}{E_{A R Q}^{\text {tot }}}\left(1-P E R_{A R Q}\right) \\
=\frac{\left(P_{t r}+P_{r e}\right) n T_{t r}}{\left(P_{t r}+P_{r e}\right)(n+\alpha+\tau+a c k) T_{t r}}\left(1-P E R_{A R Q}\right) \\
=\frac{n}{(n+\alpha+\tau+a c k)}\left(1-P E R_{A R Q}\right)
\end{gathered}
$$

where $E_{A R Q}^{e f f}$ is the energy consumed by the payload only, $E_{A R Q}^{\text {tot }}$ is the total energy consumed.

\subsubsection{FEC energy efficiency mathematical derivation}

The energy consumption of FEC is given by:

$$
E_{F E C}=E_{F E C}^{t r}+E_{F E C}^{r e}+E_{d e c}+E_{e n c}
$$

Using convolution turbo code as forward error correction techniques, encoding (Eenc) and decoding energy (Edec) are considered to be negligibly small (Sankarasubramaniam et al., 2003; Tian et al., 2008), and from Figure 1 (b), the expression for the energy efficiency is defined as:

$$
\begin{gathered}
E f f_{F E C}=\frac{E_{F E C}^{e f f}}{E_{F E C}^{t o t}}\left(1-P E R_{F E C}\right) \\
=\frac{\left(P_{t r}+P_{r e}\right)(n-k) T_{t r}}{\left(P_{t r}+P_{r e}\right)(n+\alpha) T_{t r}}\left(1-P E R_{F E C}\right) \\
=\frac{(n-k)}{(n+\alpha)}\left(1-P E R_{F E C}\right)
\end{gathered}
$$

where $P E R_{F E C}$ is calculated using equation (13).

\subsection{Simulation}

Simulation offers a powerful tool to validate mathematical analysis. The simulation is carried out for a two system using different error correction techniques using MATLAB. 
Two types of parameters are considered for design and configuration. Energy efficiency and packet probability of success (PAR) are taken as the main performance factors in comparing between the two systems.

\subsubsection{Design parameters}

The design parameters are the parameters that can be varied in order to study their effect on the system energy efficiency. In the first system ARQ technique is used as the error correction technique, where 8-PSK is used as the modulation technique as it is the best modulation technique in underwater channel as stated in the literature. In the second convolutional coding is used as the FEC error correction technique (Labrador et al., 2009).

The design parameters used are the distance, shipping factor and wind speed. Shipping factor and wind speed are taken as a representative for variable channel conditions; any other channel condition factor will have the same effect.

Modulation and encoding technique types and design parameters can be written as in Table 2. below:

\begin{tabular}{|c|c|c|}
\hline Parameter & Description & Type or Value \\
\hline Modulation & Modulation technique used in ARQ case & 8-PSK \\
\hline Encoding & Encoding technique used for error correction & Convolution coding \\
\hline Distance & Communication distance & From 800 to $3000 \mathrm{~m}$ \\
\hline Shipping factor & Factor describe the effect of shipping & From 0 to 1 \\
\hline Wind speed & Factor describe the effect of wind & Any value in $\mathrm{m} / \mathrm{s}$ \\
\hline
\end{tabular}

Table 2. Modulation, Encoding Types and Design Parameters

\subsubsection{Configuration parameters}

The simulation is carried out using MATLAB.

In the transmitter a random bit generator is used with the parameters as follows:

- $\quad$ size of signal constellation $\mathrm{M}=8$;

- Number of bit per symbol $\mathrm{k}=3$,

- $\quad$ Number of bit processed $n=3 e^{4}$.

Binary data stream are created as a column vector using the function: $x=$ randint $(n, 1)$;

A Bit-to-Symbol mapping which convert the bits in $\mathrm{x}$ into $\mathrm{k}$-bit symbols is done using the following MATLAB function:

- $\quad$ xsym = bi2de $\left(\right.$ reshape $(x, k$, length $(x) / k) .,{ }^{\prime}$, left-msb');

Then an 8-PSK modulator is used to modulate the signal with the function:

- $\quad \mathrm{y}=$ modulate (modem.pskmod (M), xsys);

The value of SNR in underwater channel is calculated as in section 2.1.3., and an AWGN function is used as: 
- $\quad$ ynoisy = awgn (y, snr, “measured');

In the receiver side 8-PSK demodulator is used to demodulate the signal using the function:

- $\quad$ zsym = demodulate (modem.pskdemod (M), noisy);

the Symbol-to-Bit mapping is done using the function:

- $\quad$ z $=$ de2bi (zsym,'left-msb');

then BER is obtained by comparing the input $\mathrm{x}$ with the output $\mathrm{z}$ using the function:

- $\quad$ [number_of_errors,bit_error_rate] = biterr $(\mathrm{x}, \mathrm{z})$;

The energy efficiency is calculated from the BER as in section 2.2.3.

In the second system the 8-PSK is replaced by a convolutional encoder with the following:

Trellis is defined using the following function:

- $\quad \mathrm{t}=$ poly2trellis $(3,[57])$;

Then puncturing is attained by the following function:

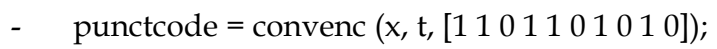

This puncturing is for $5 / 6$ code rate.

Then 0 bit is mapped to 1 and 1 bit to -1 using the function:

- $\quad$ tcode $=1-2^{*}$ punctcode;

The value of SNR in underwater channel is written as in section 2.1.3., and an AWGN function is used as:

- $\quad$ ncode = awgn(tcode, snr, 'measured');

In the receiver side, the punctured code is decoded by viterbi using the function:

- $\quad$ decoded = vitdec(ncode, t, 96, 'trunc', ...'unquant', [1 11011101010101$)$; \% Decode.

then BER is obtained by comparing the input $x$ with the output decoded using the function:

- $\quad$ [numErrPE, berPE] $=$ biterr $($ decoded, $x)$;

The energy efficiency is calculated from the BER as in section 2.2.4

\subsection{Results and analysis}

The results are obtained using a MATLAB, assuming LinkQuest UWM2000 acoustic modem (LinQuest Inc., 2011), and the parameters as given in Table 3:

First, a suitable frequency range based on AN Factor as in Figure 2 was calculated; this frequency range corresponds to the minimum AN factor. A suitable range is found from 10 $\mathrm{KHz}$ up to $25 \mathrm{KHz}$, below and above this range the AN Factor increases sharply.

From Figures 3 (a) and 3 (b), it is clear that transmission energy efficiency of both techniques increases with increasing packet size in short distances, whereas decreases in long distances 


\begin{tabular}{|c|c|c|}
\hline \multirow{2}{*}{ Symbol } & \multicolumn{2}{|c|}{ Parameters } \\
\cline { 2 - 3 } & Definition & Quantity \\
\hline$P_{t}$ & Transmitting Power & $2 \mathrm{~W}$ \\
\hline$P_{r e}$ & Receiving Power & 0.75 \\
\hline$R$ & Bit Data Rate & $10 \mathrm{kbps}$ \\
\hline$l_{a c k}$ & Acknowledge packet length & 7 Byte \\
\hline $\mathrm{a}+\tau$ & Header + FCS length & 11 Byte \\
\hline
\end{tabular}

Table 3. Simulation Parameters

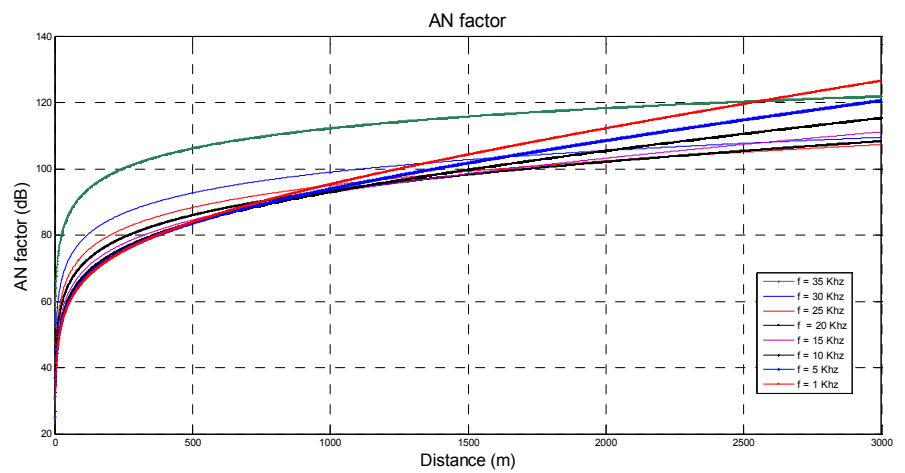

Fig. 2. AN Factor

for both techniques. It is also clear that there is only a slight differences between mathematical and simulation results which validate the results. This differences between mathematical and simulation results decreases as the number of bits transmitted in the simulation increases.

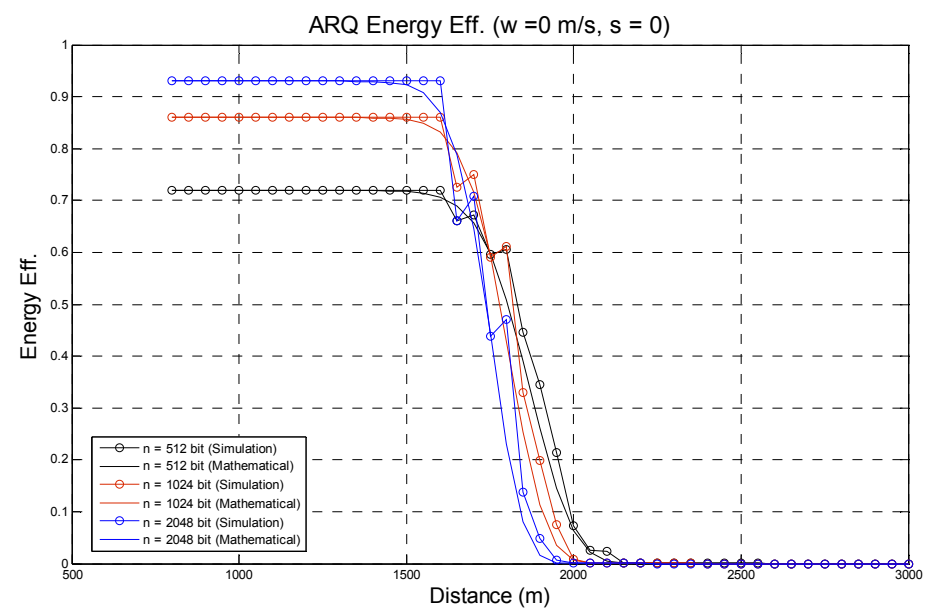

Fig. 3. (a): ARQ Transmission Energy Efficiency (Mathematical and Simulation Results) 


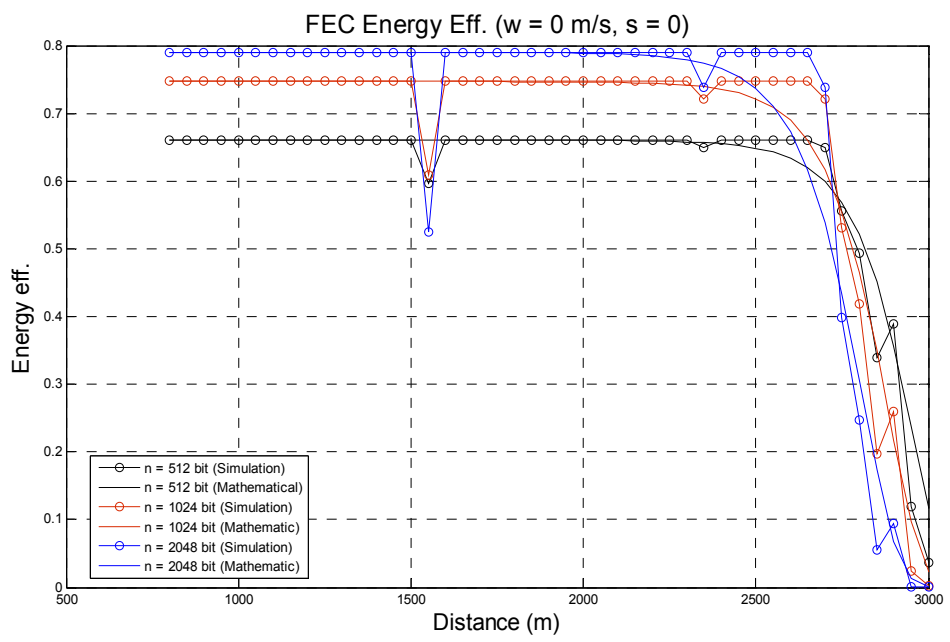

Fig. 3. (b): FEC Transmission Energy Efficiency (Mathematical and Simulation Results)

In Figure 4 (a) transmission energy efficiency of ARQ and FEC for a packet length of 512 bit is shown. It is apparent that transmission using ARQ is more energy efficient than using FEC below a specific distance (cut-off distance), and transmission using FEC is more energy efficient after this distance. The effect of shipping is unseen and can be neglected. In Figure 4 (b) the effect of wind is very clear, and the cut-off distance decreases from $1700 \mathrm{~m}$ when no wind exists to $1250 \mathrm{~m}$ when the wind speed is $1 \mathrm{~m} / \mathrm{s}$. ARQ efficiency starts to decrease at $1600 \mathrm{~m}$ when no wind exists, and at $1100 \mathrm{~m}$ when the wind speed is $1 \mathrm{~m} / \mathrm{s}$, whereas for FEC it starts to decrease at $2500 \mathrm{~m}$ when no wind exist and at $1800 \mathrm{~m}$ when the wind speed is 1 $\mathrm{m} / \mathrm{s}$.

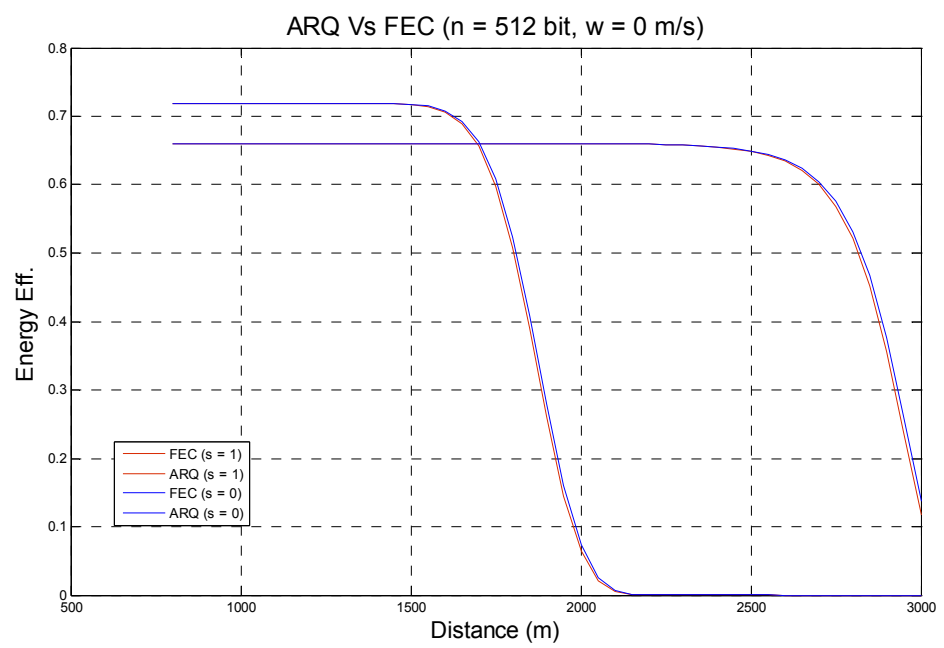

Fig. 4. (a): ARQ Vs FEC Transmission Energy Efficiency ( $\mathrm{n}=512$ bit, Variable Shipping Factor) 


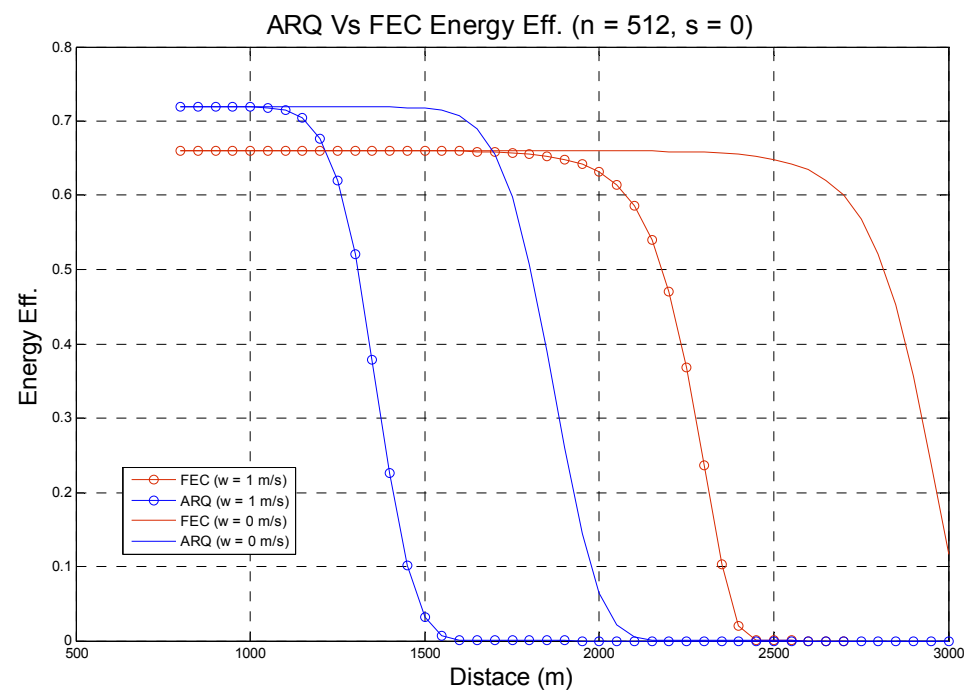

Fig. 4. (b): ARQ Vs FEC Transmission Energy Efficiency ( $n=512$ bit, Variable Wind Speed)

\subsection{Discussion}

A mathematical analysis for the energy efficiencies of ARQ and FEC data transmission has been presented. Simulation results validate the mathematical derivation results. It is found that transmission energy efficiency in underwater environment increases with increasing packet size in short distances and decreases with packet size in longer distances. It is also found that transmission using ARQ is more energy efficient below a specific distance (cutoff distance), whereas transmission using FEC is more efficient after that distance. This cutoff distance is affected by wind speed. Shipping factor has been found to have no effect on this frequency values. From those results we can say that variable distances and variable channel conditions which characterize underwater channel make it energy inefficient to use one or fixed type of error correction techniques in transmission.

The results obtained from this part will be the basis for designing and implementing a new adaptive hybrid energy efficient error correction protocol for underwater wireless sensor networks in the next part.

\section{Adaptive hybrid energy efficient error correction technique for UWSN}

As it is energy inefficient in transmission to use one or fixed type of error correction in realistic underwater conditions, it is important to consider hybrid error correction technique. This hybrid error correction technique must adapt to the variation in channel conditions and to the variation in distances between sensor nodes.

In this section, we propose an Adaptive Hybrid Energy Efficient Error Correction (AHEC) technique for Underwater Wireless Sensor Networks (UWSN) data transmission. The proposed technique depends on an adaptation algorithm which determines the most energy efficient error correction technique for the current channel conditions and distance. The 
adaptation algorithm is based on the current Bit Error Rate (BER), current error correction technique, and a pre-calculated Packet Acceptance Rate (PAR) ranges look-up table which is pre-calculated using the energy efficiency derivation has been done in the previous section. Based on this, a periodical 3-bit feedback is added to the acknowledgement packet to tell the sender which error correction technique is most suitable for current channel conditions and distance. The error correction is chosen from a pure ARQ in a good channel conditions and short distances to a hybrid of ARQ and FEC with variable encoding rates in bad channel condition and over longer distance ranges.

This section is organized as follows: the in adaptive hybrid error correction technique is presented in section 5.1. In section 5.2 we show how the pre-calculated PAR ranges look-up table is calculated. Then in section 5.3, we compare the proposed AHEC technique with the techniques that use only ARQ or only FEC as the error correction technique in variable channel conditions and over variable distance ranges.

\subsection{Adaptive hybrid error correction technique main concepts}

The results of the derivations in the previous section state that transmission energy efficiency varies with the variation in transmission distances and channel conditions for both the ARQ and the FEC. Depending on the underwater network condition and the internode distances, one technique will be better than the other. The propose AHEC technique is designed to achieve high transmission energy efficiency in such conditions, by adaptively changing the error correction technique used.

The technique works like this: for variable distances and variable channel conditions, AHEC technique always search for the technique with the highest energy efficiency, and since reliability is one part in transmission energy efficiency calculation as stated in equation (10), it will also be a reliable technique. The technique depends on an adaptation algorithm which based on the current PAR, current encoding technique used, and a pre-calculated PAR ranges look-up table to determine which error correction technique is most suitable for the current distance and current channel conditions. AHEC technique can designed as in the diagram Figure 5.

In AHEC technique, only modulation technique (i.e. ARQ) is used in good channel conditions and short distances, which means low BER. In bad channel conditions and long distances a hybrid of ARQ and variable code rates convolutional encoding are used.

Variable code rates are obtained using puncturing technique by deleting a part of the bits of low-rate convolution code (Begin et al., 1990), as in Table 4., and it is represented in

\begin{tabular}{|c|c|}
\hline Code rate & Puncturing Matrix \\
\hline $2 / 3$ & {$\left[\begin{array}{llll}1 & 1 & 0 & 1\end{array}\right]$} \\
\hline $3 / 4$ & 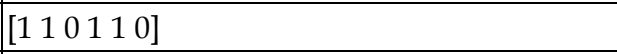 \\
\hline $4 / 5$ & {$\left[\begin{array}{llllllll}1 & 1 & 0 & 1 & 1 & 0 & 1 & 0\end{array}\right]$} \\
\hline $5 / 6$ & 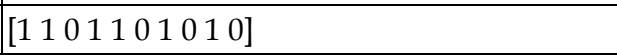 \\
\hline $6 / 7$ & {$\left[\begin{array}{llllllllllll}1 & 1 & 0 & 1 & 1 & 0 & 1 & 0 & 1 & 0 & 1 & 0\end{array}\right]$} \\
\hline
\end{tabular}

Table 4. Puncturing Matrix 


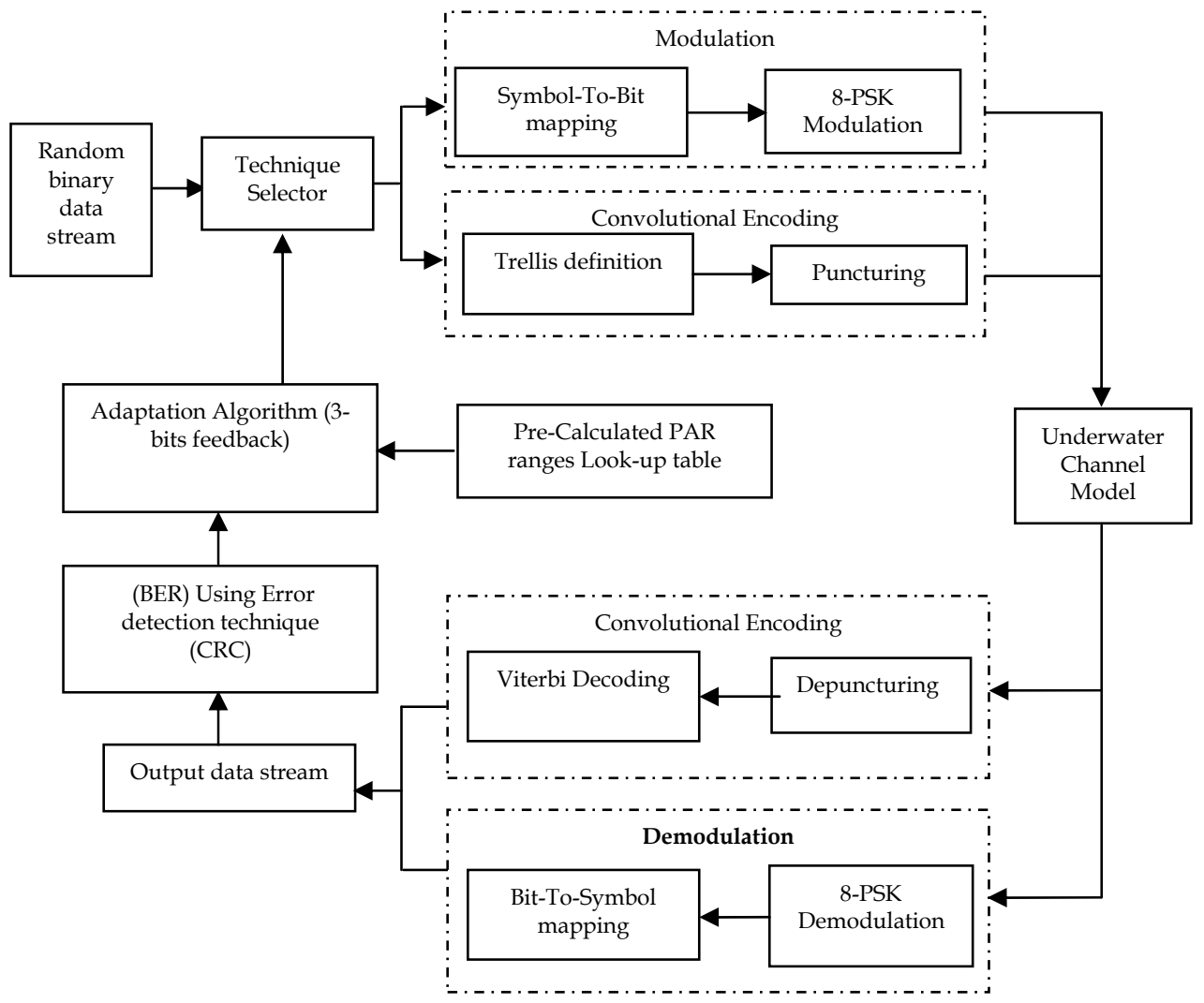

Fig. 5. AHEC Technique Design

MATLAB using systematic puncturing convolution codes with the parameters obtained from (Begin et al., 1990) as shown in Table 5.

\begin{tabular}{|l|l|l|l|l|l|}
\hline \multicolumn{1}{|c|}{$\mathrm{R}_{\mathrm{c}}$} & \multicolumn{1}{c|}{$2 / 3$} & \multicolumn{1}{c|}{$3 / 4$} & \multicolumn{1}{c|}{$4 / 5$} & \multicolumn{1}{c|}{$5 / 6$} & \multicolumn{1}{c|}{$6 / 7$} \\
\hline \multicolumn{1}{|c|}{$\mathrm{d}_{\text {free }}$} & \multicolumn{1}{c|}{3} & \multicolumn{1}{c|}{3} & \multicolumn{1}{c|}{2} & \multicolumn{1}{c|}{2} \\
\hline $\mathrm{W}_{\text {dfree }}$ & 1 & 15 & 1 & 2 & 5 \\
\hline $\mathrm{W}_{\text {dfree+1 }}$ & 10 & 104 & 36 & 111 & 186 \\
\hline $\mathrm{W}_{\text {dfree+2 }}$ & 54 & 540 & 309 & 974 & 1942 \\
\hline $\mathrm{W}_{\text {dfree+3 }}$ & 226 & 2520 & 2058 & 6815 & 16428 \\
\hline $\mathrm{W}_{\text {dfree+4 }}$ & 853 & 11048 & 12031 & 43598 & 124469 \\
\hline $\mathrm{W}_{\text {dfree+5 }}$ & 3038 & 46516 & 65754 & 263671 & 887512 \\
\hline $\mathrm{W}_{\text {dfree+6 }}$ & 10432 & 190448 & 344656 & 1536563 & 6088910 \\
\hline $\mathrm{W}_{\text {dfree+7 }}$ & 34836 & 763944 & 1755310 & 8724988 & 40664781 \\
\hline $\mathrm{W}_{\text {dfree+8 }}$ & 114197 & 3016844 & 8754128 & 46801477 & 266250132 \\
\hline
\end{tabular}

Table 5. Minimum Hamming Distances (dfree) and Weight Distribution (wdfree) for Variable Rate Convolutional Codes. 


\subsubsection{AHEC technique adaptation algorithm}

The adaptation algorithm is as follows:

Using error detection technique in the receiver, BER is periodically calculated, and from the BER, PAR is calculated using the packet length $n$ as:

$$
P A R=(1-B E R)^{n}
$$

Then the suitable error correction technique is calculated from the function:

$$
J=f(P A R, I, P A R M A X(I, J), P A R M I N(I, J))
$$

where $\mathrm{J}$ is the suitable error correction technique required, PAR is the current packet acceptance rate, I is the current error correction technique used, and PARMAX $(\mathrm{I}, \mathrm{J})$, PARMIN(I,J) are the pre-calculated PAR ranges look-up.

We can mathematically model this function as in the following formula:

$$
J=\sum_{n=1}^{6} n \times I_{A_{i}^{n}}(P A R)
$$

where $A_{i}^{n}$ is a look-up table taken from the energy efficiency derivation of six error correction techniques (One ARQ and five varying code rates FEC), and

$$
I_{B}(x)=\left\{\begin{array}{l}
1 \ldots . \text { if } . x \in B \\
0 \ldots . . . \text { otherwise }
\end{array}\right.
$$

From the value of $\mathrm{J}$ obtained, a 3-bit feedback is added to the acknowledgement to state which error correction technique to use as in Table 6 . below:

\begin{tabular}{|c|c|c|c|}
\hline Correction Technique & Consists of & FEC Code Rate & Feedback \\
\hline 1 & Pure ARQ & & 000 \\
\hline 2 & Hybrid ARQ\& FEC & $6 / 7$ & 001 \\
\hline 3 & Hybrid ARQ\& FEC & $5 / 6$ & 010 \\
\hline 4 & Hybrid ARQ\& FEC & $4 / 5$ & 011 \\
\hline 5 & Hybrid ARQ\& FEC & $3 / 4$ & 100 \\
\hline 6 & Hybrid ARQ\& FEC & $2 / 3$ & 101 \\
\hline
\end{tabular}

Table 6. Error Correction Techniques Details 


\subsection{Pre-calculated PAR ranges look-up table calculations}

The pre-calculated PAR ranges look-up table is calculated as follows:

1. Transmission energy efficiencies and PARs using six error correction techniques (One ARQ plus five variable code rates FECs) for variable values of SNR are found as in section 2.2.3 and section 2.2.4.

2. Starting with the SNR values which gives PAR values equal to 1 for all the techniques; at this SNR ARQ will have the maximum energy efficiency compared to the others, so the PAR for all those technique at this point is the maximum values in the ranges which makes the suitable technique is technique 1 (pure $A R Q$ ). This means $\operatorname{PARMAX}_{\mathrm{J}, 1}=1$, i.e. if the current technique is $J$ and the current PAR is in the range that has 1 as the maximum value, then technique one is the most energy efficient technique.

3. Then decreasing SNR value until the energy efficiency of the first technique is less than the energy efficiency of the second technique; at this SNR the PAR for all technique will be the minimum values in the ranges which makes the suitable technique is technique 1 (pure ARQ). This means the PAR of any technique $\mathrm{J}$ at this point $=\mathrm{PARMIN}_{\mathrm{J}, 1}$,i.e. if the PAR of the current technique $J$ is in between PARMIN $N_{J, 1}$ and PARMAX $X_{J 1}$, then technique one is the most energy efficient technique. As the minimum values in the first ranges equal the maximum values in the second range, then:

$$
\operatorname{PARMAX}_{\mathrm{J}, 2}=\text { PARMIN }_{\mathrm{J}, 1}
$$

Then decreasing SNR value until the energy efficiency of the second technique is less than the energy efficiency of the third technique; at this SNR the PAR for all technique will be the minimum values in the ranges which makes the suitable technique is technique number two.

This means the PAR of any technique J at this point $=$ PARMIN $_{\mathrm{J}, 2}$ i.e. if the PAR of the current technique $\mathrm{J}$ is in between PARMIN $\mathrm{J}_{\mathrm{J}, 2}$ and $\mathrm{PARMAX}_{\mathrm{J}, 2}$, then technique number 2 is the most energy efficient technique.

As the minimum values in the second ranges equal the maximum values in the third range, then:

$$
\operatorname{PARMAX}_{\mathrm{J}, 3}=\text { PARMIN }_{\mathrm{J}, 2}
$$

4. Then decreasing SNR value until the energy efficiency of the third technique is less than the energy efficiency of the fourth technique; at this SNR the PAR for all technique will be the minimum values in the ranges which makes the suitable technique is technique number 3.

This means the PAR of any technique $J$ at this point $=$ PARMIN $_{J, 3}$ i.e. if the PAR of the current technique $\mathrm{J}$ is in between PARMIN $\mathrm{J}_{\mathrm{J}, 3}$ and PARMAX $\mathrm{X}_{\mathrm{J}, 3}$, then technique number 3 is the most energy efficient technique.

As the minimum values in the third ranges equal the maximum values in the fourth range, then:

$$
\text { PARMAX }_{\mathrm{J}, 4}=\text { PARMIN }_{\mathrm{J}, 3}
$$

5. Then decreasing SNR value until the energy efficiency of the fourth technique is less than the energy efficiency of the fifth technique; at this SNR the PAR for all technique 
will be the minimum values in the ranges which makes the suitable technique is technique number 4 .

This means the PAR of any technique $\mathrm{J}$ at this point $=\mathrm{PARMIN}_{\mathrm{J}, 4}$ i.e. if the PAR of the current technique $\mathrm{J}$ is in between $\mathrm{PARMIN}_{\mathrm{J}, 4}$ and $\mathrm{PARMAX}_{\mathrm{J}, 4}$, then technique number 4 is the most energy efficient technique.

As the minimum values in the fourth ranges equal the maximum values in the fifth range, then:

$$
\text { PARMAX }_{\mathrm{J}, 5}=\text { PARMIN }_{\mathrm{J}, 4}
$$

6. Then decreasing SNR value until the energy efficiency of the fifth technique is less than the energy efficiency of the six technique; at this SNR the PAR for all technique will be the minimum values in the ranges which makes the suitable technique is technique 5 .

This means the PAR of any technique $\mathrm{J}$ at this point $=\mathrm{PARMIN}_{\mathrm{J}, 5}$ i.e. if the PAR of the current technique $\mathrm{J}$ is in between PARMIN $\mathrm{J}_{\mathrm{J}, 5}$ and PARMAX $\mathrm{J}_{\mathrm{J}, 5}$, then technique number 5 is the most energy efficient technique.

As the minimum values in the fifth ranges equal the maximum values in the sixth range, then:

$$
\operatorname{PARMAX}_{\mathrm{J}, 6}=\text { PARMIN }_{\mathrm{J}, 5}
$$

7. At last zero will be the minimum values for the ranges that makes technique six is the most energy efficient technique (PARMIN $\mathrm{J}_{\mathrm{J}, 6}=0$, for all techniques).

\subsection{Results and discussion}

In this section we first present how to calculate the pre-calculated PAR ranges look-up table, which is an essential part in our adaptation algorithm, then we will compare our AHEC technique with the previous works in the literature that depend on only ARQ or only FEC for error correction (Lee et al., 2008; Gao et al., 2009; Tan et al., 2003; Xie and Cui, 2007) in variable channel conditions and variable distances.

\subsubsection{AHEC technique transmission energy efficiency calculations}

To calculate the pre-calculated PAR ranges look-up table, energy efficiencies versus PARs for the six techniques are calculated as in section 2.2.3 for ARQ and in section 2.2.4 for the five variable code rate FEC, then the pre-calculated PAR ranges look-up table can be calculated as in section 3.3, and it can be displayed as in Table 7 below:

\begin{tabular}{|c|c|c|c|c|c|c|}
\hline $\mathrm{i} \backslash \mathrm{j}$ & 1 & 2 & 3 & 4 & 5 & 6 \\
\hline 1 & $0.95-1.0$ & $0.95-0.0$ & & & & \\
\hline 2 & 1.0 & $0.89-1.0$ & $0.84-0.89$ & $0.62-0.84$ & $0.32-0.62$ & $0.00-0.32$ \\
\hline 3 & 1.0 & $0.92-1.0$ & $0.89-0.92$ & $0.72-0.89$ & $0.45-0.72$ & $0.00-0.45$ \\
\hline 4 & 1.0 & $0.96-1.0$ & $0.94-0.96$ & $0.85-0.94$ & $0.68-.85$ & $0.00-0.68$ \\
\hline 5 & 1.0 & $0.98-1.0$ & $0.97-0.98$ & $0.92-0.97$ & $0.81-0.92$ & $0.00-0.81$ \\
\hline 6 & 1.0 & $0.995-1.0$ & $0.992-0.995$ & $0.992-0.98$ & $0.95-0.98$ & $0.00-0.95$ \\
\hline
\end{tabular}

Table 7. Pre-Calculated Look-Up PAR Ranges Table 
From the Pre-calculated PAR ranges look-up table above, and from the current PAR, current encoding technique, AHECT energy efficiency can be calculated as in section 3.2

\subsubsection{Transmission using AHEC technique versus the transmission using $A R Q$ and FEC transmission energy efficiency}

Figure 6 below gives a comparison between the energy efficiency of transmission using AHEC technique and the transmission using pure ARQ and pure FEC for varying distances.

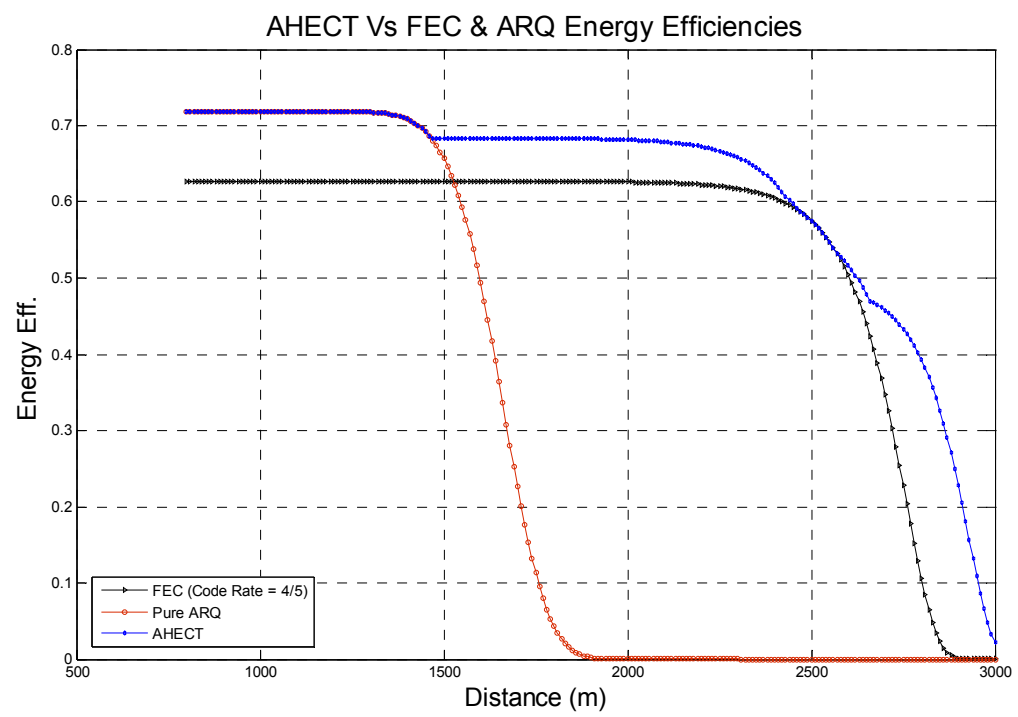

Fig. 6. AHEC Technique Vs ARQ \& FEC Energy Efficiency (Variable Distances Case)

From this figure it is clear that transmission using AHEC technique is more energy efficient than using both ARQ and FEC in variable distances situation.

Compared with the pure $\mathrm{ARQ}$, transmission using AHEC technique achieves $10 \%$ increase in saving energy when the distance is around $1500 \mathrm{~m}$ and more than $60 \%$ when the distance increases above $1700 \mathrm{~m}$. When compared with transmission using FEC, it achieves around $10 \%$ increase in energy saving when the distance is below $1500 \mathrm{~m}$, and around $7 \%$ saving when the distance goes above $1500 \mathrm{~m}$.

In Figure 7; variable wind speed is taken as a measure for the variation in channel conditions. From this Figure it is clear that transmission using AHEC technique is more energy efficient than both techniques using ARQ and FEC for variable wind speed (i.e. variable channel conditions). Compared with the pure $A R Q$, and when the transmission distance is $1500 \mathrm{~m}$, transmission using AHEC technique achieves $6 \%$ increase in energy saving when wind speed is $0.5 \mathrm{~m} / \mathrm{s}$, more than $50 \%$ energy saving when wind speed 


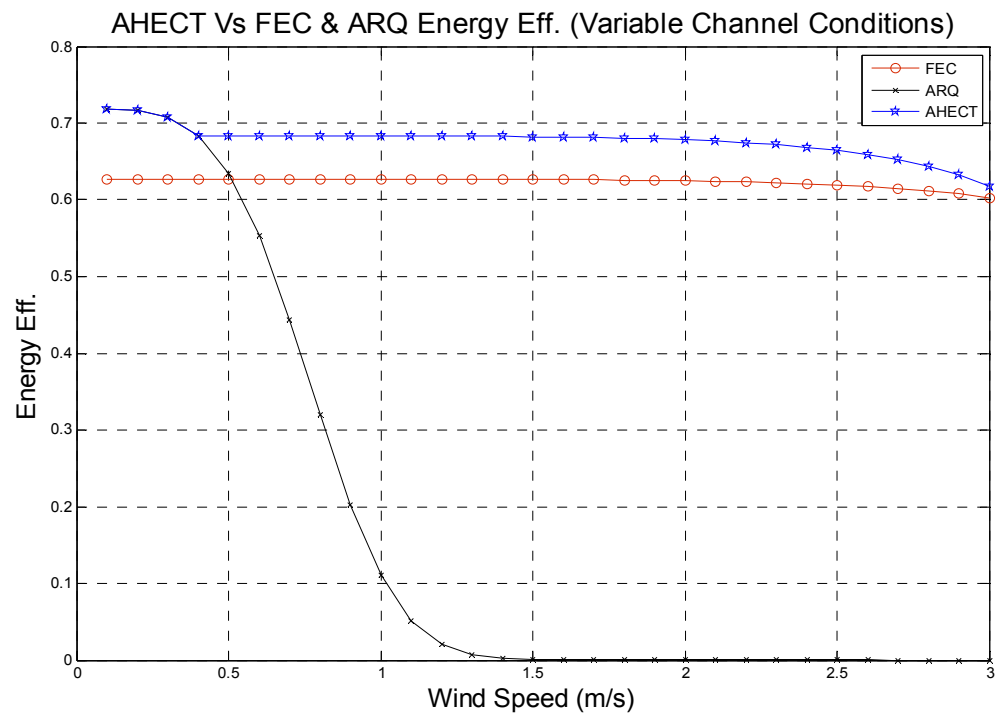

Fig. 7. AHEC Technique Vs ARQ \& FEC Transmission Energy Efficiency (Variable Channel Conditions)

increases to $1 \mathrm{~m} / \mathrm{s}$, and more than $60 \%$ when wind speed is greater than $1.5 \mathrm{~m} / \mathrm{s}$. When compared with transmission using FEC, transmission using AHEC technique achieves around $8 \%$ increase in energy saving when wind speed is below $0.5 \mathrm{~m} / \mathrm{s}$ and around $6 \%$ when wind speed is more than $0.5 \mathrm{~m} / \mathrm{s}$.

\section{Conclusions}

Underwater wireless sensor network (UWSN) is a promising engineering endeavour which will ensure progress in monitoring and exploiting the ocean's vast resources. But until now it faces many challenges, the most important of which is the severe energy constraint of the batteries, which cannot be recharged or replaced in aquatic medium. Complicating the issue is the variability of the channel conditions and the distances between underwater sensors.

In this chapter, we have mathematically analyzed the transmission energy efficiency for two main error correction techniques, ARQ and FEC, in underwater environment. A simulation is done to validate the mathematical derivation results. Transmission using ARQ is found to be more energy efficient than transmission using FEC below specific distances, and transmission using FEC is found to be better after that. We call this specific distance the cutoff distance. We found that this cut-off distance is not fixed and varies with the variation in channel conditions and packet size.

Based on the mathematical analysis, we have proposed an energy efficient Adaptive Hybrid Error Correction (AHEC) technique for transmission. The proposed technique adaptively 
changes the error correction technique to the technique with the highest transmission energy efficiency compared to the others. An adaptation algorithm which based on the current packet acceptance rate (PAR), current encoding technique, and a pre-calculated PAR ranges look-up table has been proposed. From the output of the adaptation algorithm, a periodic 3bit feedback is sent to the sender indicating which error correction technique is most suitable given the current distance and channel conditions. The proposed technique has been compared with techniques that use only ARQ or FEC. The results show that our proposed technique is more energy efficient than either of them.

\section{References}

Akyildiz, I. Pompili, D. and Melodia, T. (2004). Challenges for Efficient Communication in Underwater Acoustic Sensor Networks, ACM Sigbed Review, Vol. 1, 2004.

Akyildiz, I. Pompili, D. and Melodia, T. (2005). Underwater acoustic sensor networks: research challenge, Ad Hoc Networks (Elsevier), Vol. 3, pp. (257-279), 2005.

Begin, G. Haccoun, D. and Paquin, C. (1990). Further Results on High - Rate Puncturing G Convolutional codes for viterbi and sequential decoding, IEEE transaction on Communications, Vol. 38, pp. (1922-1928), 1990.

Bin, L. Garcin, F. Ren, F. and Lin, C. (2008). A study of forward error correction schemes for reliable transport in underwater sensor networks, Proceedings of the 5th Annual IEEE Communications Society Conference on Sensor, Mesh and Ad Hoc Communications and Networks (SECON '08), pp (197-205), 2008.

Brekhovskikh, L. and Lysanov, Y. (2003). Fundamental of ocean acoustics: Springer, 1982.

Colin Y., Chan, and Motani M. (2007). An integrated energy efficient data retrieval protocol for underwater delay tolerant networks, Proceeding of IEEE ocean'07, Aberdeen, Scotland, 2007.

Gao, M. Soh, W. and Tao, M. (2009). A transmission scheme for continuous ARQ protocols over underwater acoustic channels, Proceeding of the 2009 IEEE international conference on Communications (ICC'09), 2009.

Guo, Z. Xie, P. Cui, J. and Wang, B. (2006). On applying network coding to underwater sensor networks, Proceedings of ACM WUWNet'06, Los Angeles, CA, 2006.

Harris, A. and Zorzi, M. (2007). Modelling the underwater acoustic channel in ns2, Proceedings of the 2nd international conference on Performance evaluation methodologies and tools, 2007.

Joshy, S. and Babu, A. (2010). Capacity of Underwater Wireless Communication Channel with Different Acoustic Propoagation Loss Models, International Journal of Computer Networks \& Communications (IJCNC), Vol. 2, 2010.

Kunal K., Tripathi R. and Singh V. (2010). An HARQ based Optimized Error Correction Technique, International Journal of Computer Applications, Vol. 9, 2010.

Labrador, Y. Karimi, M. Pan, D. And Miller, J. (2009). Modulation and error correction in the underwater acoustic communication channel, International Journal of Computer Science and Network Security, Vol. 9, pp. (123-130), 2009.

Lee J. Kim J. Lee J. Jang Y. and Dho K. (2008). An improved ARQ scheme in underwater acoustic sensor networks, Proc. MTS/IEEE Oceans, Kobe, Japan, 2008, pp. 1-5. 
Lee, W. Kim, J. Lee, J. Jang, Y and Dho, K. (2008). An improved ARQ scheme in underwater acoustic sensor networks, Proceeding of MTS/IEEE Oceans, Kobe, Japan, pp. (1-5), 2008.

LinkQuest Inc. Underwater Acoustic Modem Models. June 2010 (Available: http:/ / www.link-quest.com)

Liu, B. Chen, H. Lei, X. Ren, F. and Sezaki , K. (2010). Internode distance-based redundancy reliable transport in underwater sensor networks, EURASIP J. Wireless Comm. and Networking, 2010.

Lin S., Costello D., Miller M., (1984) Automatic-Repeat-Request Error-Control Schemes. IEEE Communications Magazine. pp (5-17), 1984.

Pompili, D. (2007). Efficient communication protocols for underwater acoustic sensor networks," $\mathrm{PhD}$, School of Electrical and Computer Engineering, Georgia Institute of Technology, 2007.

Preisig, J. (2007). Acoustic Propagation considerations for underwater Acoustic communications network Development, SIGMOBILE Mob. Computer Commun. Rev., Vol. 11, pp. (2-10), 2007.

S. Joshy and A. Babu, "Capacity of Underwater Wireless Communication Channel with Different Acoustic Propoagation Loss Models," International Journal of Computer Networks \& Communications (IJCNC), vol. 2, 2010.

Sankarasubramaniam, Y. Akyidiliz, I. and Mclaughlin, S. (2003). Energy efficiency based packet size optimization in wireless sensor networks, Proceeding of the 1st IEEE International Workshop on Sensor Network Protocols and Applications SNPA'03 (held in conjunction with IEEE ICC'03), Anchorage, Alaska, USA, 2003.

Stojanovic, M. and Preisig. J. (2009). Underwater Acoustic Communication Channels: Propagation Models and Statistical Characterization, IEEE Communications, pp. 84-89, 2009.

Tan H., Seah W. and Doyle L. (2007). A multi-hop ARQ protocol for underwater acoustic networks, Proceedings of OCEANS 2007 , Europe, 18-21 June 2007 pp. 1 - 6.

Tian, Z. Yan, D. and Liang, Q. (2008). Energy efficiency analysis of error control schemes in wireless sensor networks, Proceeding of International Wireless Communications and Mobile Computing Conference, IWCMC '08, pp. (401-405), 2008.

Urick R. J. (1983). Principles of Underwater Sound, McGraw-Hill, New York.

Valera, A. Lee, P. Tan, H. (2009). Implementation and Evaluation of Multihop ARQ for Reliable Communications in Underwater Acoustic Networks, Proceedings of the IEEE OCEANS Conference, Bremen, Germany, 2009.

Webb, S. (1992). The equilibrium oceanic microseism spectrum, J. Acoust. Soc., Vol. 92, pp. (2141-2158), 1992.

Wenz and Gordon, M. (1936). Acoustic Ambient Noise in the Ocean: Spectra and Sources, The Journal of the Acoustical Society of America, Vol. 34, 1936.

Xie P. (2007). Underwater acoustic sensor networks: Medium access control, routing and reliable transfer, 2007.

Xie, P. and Cui, J. (2007). An FEC- based reliable data transport protocol for Underwater sensor networks, Proc. of 16th International Conference on Computer Communications and Networks, pp.(747-753), 2007. 
Yang H. and Liu B. (2009). Optimization of Energy Efficient Transmission in Underwater Sensor Networks, Proceedings of CoRR, 2009. 


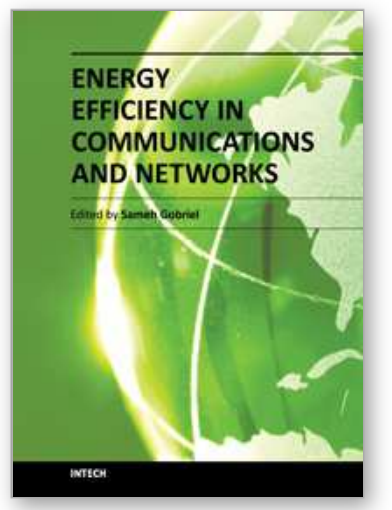

\author{
Energy Efficiency in Communications and Networks \\ Edited by Dr. Sameh Gobriel
}

ISBN 978-953-51-0482-7

Hard cover, 142 pages

Publisher InTech

Published online 04, April, 2012

Published in print edition April, 2012

The topic of "Energy Efficiency in Communications and Networks" attracts growing attention due to economical and environmental reasons. The amount of power consumed by information and communication technologies (ICT) is rapidly increasing, as well as the energy bill of service providers. According to a number of studies, ICT alone is responsible for a percentage which varies from $2 \%$ to $10 \%$ of the world power consumption. Thus, driving rising cost and sustainability concerns about the energy footprint of the IT infrastructure. Energyefficiency is an aspect that until recently was only considered for battery driven devices. Today we see energyefficiency becoming a pervasive issue that will need to be considered in all technology areas from device technology to systems management. This book is seeking to provide a compilation of novel research contributions on hardware design, architectures, protocols and algorithms that will improve the energy efficiency of communication devices and networks and lead to a more energy proportional technology infrastructure.

\title{
How to reference
}

In order to correctly reference this scholarly work, feel free to copy and paste the following:

Ammar Babiker and Nordin Zakaria (2012). Energy Efficient Communication for Underwater Wireless Sensors Networks, Energy Efficiency in Communications and Networks, Dr. Sameh Gobriel (Ed.), ISBN: 978-953-510482-7, InTech, Available from: http://www.intechopen.com/books/energy-efficiency-in-communications-andnetworks/energy-efficient-communications-for-underwater-wireless-sensors-networks

\section{INTECH}

open science | open minds

\section{InTech Europe}

University Campus STeP Ri

Slavka Krautzeka 83/A

51000 Rijeka, Croatia

Phone: +385 (51) 770447

Fax: +385 (51) 686166

www.intechopen.com

\section{InTech China}

Unit 405, Office Block, Hotel Equatorial Shanghai

No.65, Yan An Road (West), Shanghai, 200040, China

中国上海市延安西路65号上海国际贵都大饭店办公楼 405 单元

Phone: +86-21-62489820

Fax: $+86-21-62489821$ 
(C) 2012 The Author(s). Licensee IntechOpen. This is an open access article distributed under the terms of the Creative Commons Attribution 3.0 License, which permits unrestricted use, distribution, and reproduction in any medium, provided the original work is properly cited. 